\title{
Genetically Engineered Bacterial Protein Nanoparticles for Targeted Cancer Therapy
}

This article was published in the following Dove Press journal:

International Journal of Nanomedicine

\author{
Haiyan Yang' \\ Fujie Jiang' \\ Xiaojuan $\mathrm{Ji}^{2}$ \\ Lu Wang' \\ Yaotai Wang' \\ Liang Zhang ${ }^{3}$ \\ Yu Tang' \\ Disen Wang' \\ Yong Luo' \\ Ningshan $\mathrm{Li}^{\mathrm{I}}$ \\ Qi Wang' \\ Jianzhong Zou' \\ 'State Key Laboratory of Ultrasound in \\ Medicine and Engineering, College of \\ Biomedical Engineering, Chongqing \\ Medical University, Chongqing 400016, \\ China; Chongqing Key Laboratory of \\ Biomedical Engineering, Chongqing \\ Medical University, Chongqing 400016, \\ People's Republic of China; ${ }^{2}$ Department \\ of Ultrasound, Children's Hospital of \\ Chongqing Medical University, Ministry of \\ Education Key Laboratory of Child \\ Development and Disorders; National \\ Clinical and Research Center of Child \\ Health and Disorders; China \\ International Science and Technology \\ Cooperation Base of Child Development \\ and Critical Disorders; Chongqing \\ Engineering Research Center of Stem \\ Cell Therapy, Chongqing 400016, \\ People's Republic of China; ${ }^{3}$ Institute of \\ Ultrasound Imaging, The Second \\ Affiliated Hospital of Chongqing Medical \\ University, Chongqing 4000 I0, People's \\ Republic of China
}

Correspondence: Jianzhong Zou State Key Laboratory of Ultrasound in Medicine and Engineering, College of Biomedical Engineering, Chongqing

Medical University, Chongqing, Yuzhong

District 400016, People's Republic of

China

Tel +86-13708302390

Email zoujzh@cqmu.edu.cn
Purpose: Cancer treatment still faces big challenges in the clinic, which is raising concerns over the world. In this study, we report the novel strategy of combing bacteriotherapy with high-intensity focused ultrasound (HIFU) therapy for more efficient breast cancer treatment. Methods: The acoustic reporter gene (ARG) was genetically engineered to be expressed successfully in Escherichia coli (E. coli) to produce the protein nanoparticles-gas vesicles (GVs). Ultrasound was utilized to visualize the GVs in E. coli. In addition, it was injected intravenously for targeted breast cancer therapy by combing the bacteriotherapy with HIFU therapy.

Results: ARG expressed in E. coli can be visualized in vitro and in vivo by ultrasound. After intravenous injection, E. coli containing GVs could specifically target the tumor site, colonize consecutively in the tumor microenvironment, and it could obviously inhibit tumor growth. Meanwhile, E. coli which contained GVs could synergize HIFU therapy efficiently both in vitro and in vivo as the cavitation nuclei. Furthermore, the tumor inhibition rate in the combination therapy group could be high up to $87 \%$ compared with that in the control group.

Conclusion: Our novel strategy of combing bacteriotherapy with HIFU therapy can treat breast cancers more effectively than the monotherapies, so it can be seen as a promising strategy.

Keywords: acoustic reporter gene, gas vesicles, targeted cancer therapy, bacteriotherapy, high-intensity focused ultrasound

\section{Introduction}

Cancer therapeutic remains a big challenge in the clinic. The traditional methods of anticancer therapeutics including surgery, chemotherapy, radiotherapy, immunotherapy, and bacteriotherapy have raised the hope for cancer treatment. ${ }^{1}$ However, there are inevitable side effects including invasiveness, high toxicity, immune system destruction, drug resistance, high costs, and low treatment efficiency. ${ }^{2-4}$ In recent decades, high-intensity focused ultrasound (HIFU) has shown great potential in clinical therapy of noninvasive solid tumors. ${ }^{5-7}$ However, the practical application of HIFU for combating cancers is still limited due to its insufficient therapeutic effect. $^{8}$

To obtain better cancer therapy efficiency through HIFU ablation, synergistic agents (SAs) have recently attracted tremendous attention. Various SAs (eg, gas-filled nanoparticles, lipiodol, hydroxyapatite, and liquid fluorocarbon nanoparticles) are explored to synergize HIFU ablation for tumors by cavitation effect or changing the acoustic properties of the tumor tissue..$^{9-13}$ Those traditional SAs have been exploited to 
improve the efficiency for cancer therapy, but the cancer therapy efficiency needs to improve urgently due to i) lack of satisfying tumor-targeting ability; ii) high toxicity; iii) complex preparation process; iv) big size; v) instability; vi) short halftime in blood circulation, and those limitations have led to the unsatisfied efficiency of cancer therapy.

Live tumor-targeting bacteria vectors as the natural "microrobot" raises the new hope for cancer therapy. ${ }^{14-17}$ Many kinds of obligate or facultative anaerobic bacteria, such as Bifidobacterium, ${ }^{18,19}$ Salmonella typhimurium (S. typhimurium) ${ }^{20-22}$ Escherichia coli (E. coli $),{ }^{23-25}$ Listeria $^{26}$ and Clostridium, ${ }^{27,28}$ not only possess inherent specifically tumor-targeting activities and anti-tumor efficacy but also can be engineered to target and deliver antitumor agents for cancer therapy via genetic programs or sophisticated bio-syntheses. $^{29}$

To overcome the drawbacks of adding conventional SAs to synergize HIFU ablation for tumors, our team attempts to explore the biological targeting synergistic agents, which utilize the live tumor-targeting bacteria as the vectors to realize more efficient cancer treatment. Our preliminary experiments found that the anaerobic bacterium Bifidobacterium longum (B. longum) was able to specifically target solid tumors after intravenous (i.v.) injection B. longum which served as a vehicle can conjugate and deliver more SAs to the tumor site, synergizing HIFU efficiently for more effective cancer therapy. ${ }^{30-34}$ But this strategy still has some limitations including i) drawbacks of conventional SAs; ii) just connecting SAs to the bacterial surface of B. longum. How to further improve cancer therapy efficiency is crucial, while there is a big challenge to achieve high-efficiency cancer therapy.
Acoustic reporter gene (ARG) is the genetic constructs firstly reported by Mikhail Shapiro ${ }^{35}$ and could be expressed in E. coli and S. typhimurium. ARG expressed in bacteria could be visualized in vitro and in vivo by ultrasound for it encodes the production of gas vesicles (GVs). ${ }^{36-39} \mathrm{GVs}$, a unique class of protein-shelled nanoparticles with gasfilled in the interior, is comprised of a 2-nm-thick protein shell with dynamic sizes of typical widths from 45 to 250 $\mathrm{nm}$ and lengths from 100 to $600 \mathrm{~nm}$. The special structure of GVs allows dissolved gases to permeate in and out freely and exclude water. ${ }^{40,41}$ Especially, GVs is capable of nucleating cavitation both in their purified form and inside genetically engineered bacteria. $^{42}$ In addition, $E$. coli, as the anaerobic bacteria, can target and colonize in tumor regions after i.v. injection, and quickly be cleared from normal organs. $^{43}$ Thus, E. coli shows good biosafety. More importantly, E. coli, as the anticancer agents, can inhibit tumor growth by expressing chemotactic receptors to respond to chemotaxis towards molecular signals in the tumor microenvironment. ${ }^{44,45}$ Therefore, the strategy of utilizing GVs expression in E. coli as the new SA to synergize HIFU ablation for tumors may be an alternative option for enhancing cancer treatment.

To achieve this goal, we report a new approach to treat cancers with profound therapeutic efficacy by combing bacteriotherapy with HIFU therapy. As shown in Figure 1, we firstly genetically engineered the ARG to express in E. coli BL21(AI) to produce GVs in vitro, and then injected $E$. coli BL21(AI) which contains GVs (hereby named GVs-E. coli) into the tumor-bearing mice to inhibit the tumor growth; finally, we treated the breast cancer by HIFU therapy, tumor volume measurements and

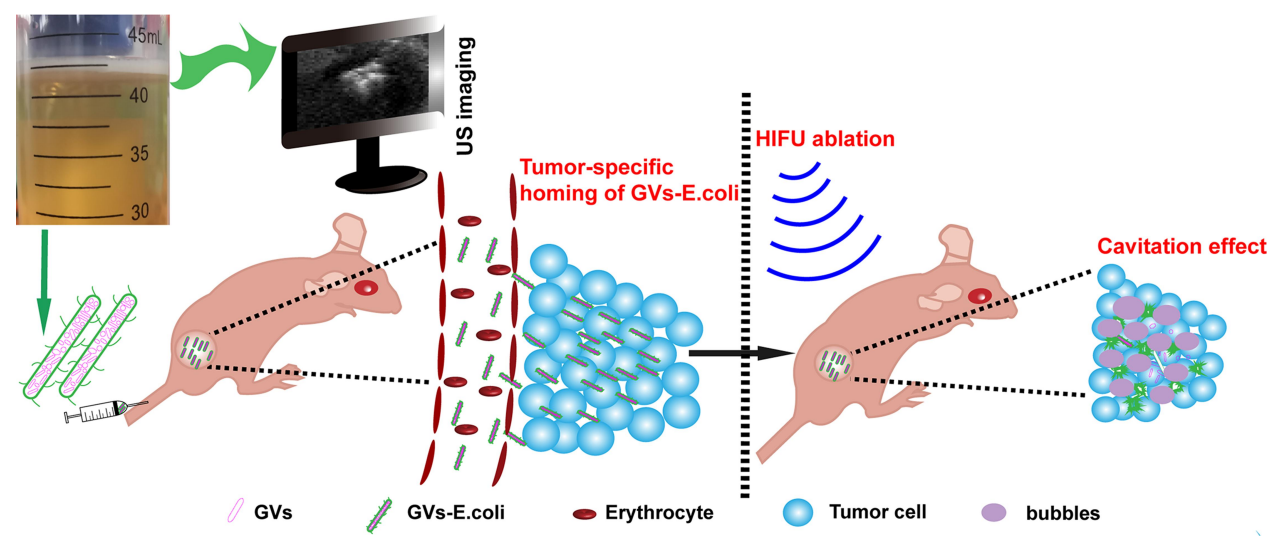

Figure I Schematic illustration of the GVs-E. coli for breast cancer therapy combing HIFU therapy and bacteriotherapy. Abbreviations: GVs, gas vesicles; GVs-E. coli, E. coli BL2I(AI) which contains GVs; HIFU, high-intensity focused ultrasound. 
histological analyses were used to evaluate the treatment efficiency of breast cancer.

\section{Materials and Methods Materials}

E. coli BL21(AI) was purchased from Shanghai Weidi Biotechnology Co., Ltd (China) and stored at $-80^{\circ} \mathrm{C}$ before use, the pET28a_T7-ARG1 was a gift from Mikhail Shapiro (Addgene plasmid \# 106473). The pQE3_green fluorescent protein (GFP) was a gift from the key laboratory of molecular biology on infection diseases, Chongqing medical university. Luria-Bertani (LB) was purchased from Hopebiol Co., Ltd (China). All chemicals were analytical grade and used without further purification.

\section{Methods}

\section{Bacteria Culture}

For bacterial culture, E. coli $\mathrm{BL} 21(\mathrm{AI})$ was cultured in $\mathrm{LB}$ liquid medium in a shaking incubator $\left(37^{\circ} \mathrm{C}, 220 \mathrm{rpm}\right)$. Bacterial concentration was estimated spectrophotometrically by determining absorbance at $600 \mathrm{~nm}$, and cell numbers were verified by plating dilutions of inoculum onto LB agar plate $\left(1 \mathrm{OD} \approx 1.06 \times 10^{9} \mathrm{CFU} / \mathrm{mL}\right)$. For expression of ARG and GFP in E. coli BL21(AI), the method was adopted from Mikhail Shapiro ${ }^{35}$ and previously described respectively, ${ }^{46}$ and the GVs-E. coli was observed directly by transmission electron microscopy (TEM, Hitachi 7500, Japan) and ultrasound using MyLab 90 (Esaote, Italy). Thereafter, E. coli BL21(AI) with or without plasmid was collected by centrifugation $\left(5000 \mathrm{rpm},-20^{\circ} \mathrm{C}, 10 \mathrm{~min}\right)$ and diluted with sterile PBS for further experiments.

\section{Cell Culture}

The human breast cancer MDA-MB-231 cells were purchased commercially from the Chinese Academy of Sciences Cell Bank (China). The cells were cultured according to ATCC recommended conditions at $37^{\circ} \mathrm{C}$ with $5 \% \mathrm{CO}_{2}$ in DMEM supplemented with $10 \%$ FBS and $1 \%$ streptomycin/penicillin.

\section{Animals and Model Establishment}

All the animal experiments and procedures were approved by the Institutional Animal Care and Use Committee at Chongqing Medical University and performed under their guidelines. Female BALB/c nude mice (20-25 g) and female $\mathrm{BALB} / \mathrm{c}$ mice (6 to 8 weeks) were supplied by the Laboratory Animal Center of Chongqing Medical University and were housed to drink and eat freely. To establish the tumor model, $0.1 \mathrm{~mL}$ PBS solutions $\left(1 \times 10^{6}\right.$ cells $)$ were subcutaneously injected into the left flank of nude mice. The volume of the tumor was calculated as $[0.5 \times$ length $\times$ (width) $)^{2}$ ]. After the tumor diameter reached $\sim 0.5 \mathrm{~mm}$, all experimental animals were randomly assigned to six groups in the following experiments. The biosafety of E. coli $\mathrm{BL} 21(\mathrm{AI})$ was evaluated using $\mathrm{BALB} / \mathrm{c}$ mice.

\section{Evaluation of Safety in vivo}

Six groups ( $\mathrm{n}=5$ per group) of healthy $\mathrm{BALB} / \mathrm{c}$ mice ( $\sim 20$ g, 4-6 weeks) were used to assess the in vivo toxicity. $1 \times 10^{8} \mathrm{CFU}$ live GVs-E. coli (selected from a series of safety experiments including different doses, data not shown) in a total volume of $100 \mu \mathrm{L}$ phosphatebuffered saline (PBS) solution was injected into mice intravenously (i.v.) in all experiments. The weight of mice was monitored every 2 days over 14 days. Blood samples were collected before (control group) and after 30 minutes, 1, 3, 7 and 14 days feeding post-injection for biochemical examinations of complete blood count (white blood cell, WBC; red blood cell, RBC; platelets, PLT; hemoglobin, HGB; mean corpuscular hemoglobin, $\mathrm{MCH}$; mean corpuscular volume, MCV), liver functional markers (alanine aminotransferase, ALT; aspartate transaminase, AST), myocardial enzymogram (creatine kinase, CK; L-lactate dehydrogenase, LDH-L), and kidney functional marker (blood urea nitrogen, BUN; creatinine, CREA). The major organs (heart, liver, spleen, lung, and kidney) were stained with H\&E for histological analysis at the corresponding time point.

\section{Tumor-Targeting Ability of E. coli BL2I(AI)}

Before and after injecting i.v. GVs-E. coli at the dose of 1× $10^{8} \mathrm{CFU} / 100 \mu \mathrm{L}$ at the $1 \mathrm{st}, 3 \mathrm{rd}, 7 \mathrm{th}$, and 14 th day, the major organs (heart, liver, spleen, lung, kidney, and tumor) of mice were extracted at the desired time points, weighed, and homogenized at $4{ }^{\circ} \mathrm{C}$ in sterile PBS. Those samples were diluted (10-fold, 100-fold, 1000-fold, etc) and spread on LB plates. After 8-24 hours of incubation, the number of GVs-E. coli colonies were counted. The bacterial titer (CFU per gram of tissue) was calculated with colony counts and tissue weights. Single colony was further picked for gram staining. 


\section{Ultrasound Imaging}

For in vitro ultrasound imaging, two groups were divided as follows: E. coli and GVs-E. coli. The ultrasound images under B mode and contrast-enhanced ultrasonography (CEUS) mode were acquired using MyLab 90 (Esaote, Italy). The gray value was measured by DFY software (Chongqing, China).

For tumor ultrasound imaging, MDA-MB-231 tumorbearing nude mice were intratumorally injected with 100 $\mu \mathrm{L}\left(1 \times 10^{8} \mathrm{CFU}\right)$, images of the tumor region were obtained by ultrasound, and the quantitative analysis of gray value was measured.

\section{Synergistic Effect of GVs-E. coli for HIFU Therapy}

A Model-JC200 Focused Ultrasound Tumor Therapeutic System (Chongqing Haifu Medical Technology Co., Ltd., Chongqing, China) was used for all HIFU experiments. The focal length, diameter, and operating frequency were 100-250 mm, 100-300 mm, and 0.5-2 MHz, respectively. A high-energy US beam was emitted from the therapeutic transducer, while changes in the targeted tissue were monitored by the diagnostic transducer in real-time.

A passive cavitation detector (PCD) was used to detect the cavitation characteristics under atmospheric pressure to compare cavitation behavior during the process of HIFU exposure. Acoustic signal from ex vivo bovine liver and tumors was recorded by the high-speed data acquisition card. Then the signal was programmed to get the corresponding spectrum 90 with fast Fourier transform on the LabView development platform (step length $15 \mathrm{~ms}$ ). The broadband noise was calculated after the band-pass filter $(5 \mathrm{MHz})$ and a band-stop filter (filter passband harmonic).

We used fresh ex vivo bovine livers to evaluate in vitro HIFU ablation efficiency. In brief, $0.1 \mathrm{~mL}$ solutions of $1 \times$ $10^{8} \mathrm{CFU} / \mathrm{mL}$ (PBS, E. coli, and GVs-E. coli) were injected into the bovine livers respectively. Immediately after that, the injection site was treated with HIFU ablation at different power $(120 \mathrm{~W}, 150 \mathrm{~W}$, and $180 \mathrm{~W})$ for $5 \mathrm{~s}$. The emitted signals were recorded with a PCD at the same time. The gray value and coagulative necrosis volume of the ablation area were quantitatively analyzed.

For in vivo HIFU synergistic therapy evaluation, MDA-MB-231 tumor-bearing nude mice (PBS+HIFU, E. coli + HIFU, GVs-E. coli + HIFU) were treated with $0.1 \mathrm{~mL}$ solutions at $1 \times 10^{8} \mathrm{CFU} / \mathrm{mL}$ respectively. 7 days after i.v. injection, the tumor site was ablated by HIFU $(150 \mathrm{~W}, 5 \mathrm{~s})$ and detected the cavitation characteristics by PCD. The 2\% 2,3,5-triphenyltetrazolium chloride (TTC) solution was used to stain the tumor tissues after $24 \mathrm{~h}$ postHIFU ablation. Gray-scale of the targeted area was automatically compared by the Gray Val 1.0 software affiliated with the HIFU device, coagulative necrosis volume (V) was calculated according to the formula: $\mathrm{V}\left(\mathrm{mm}^{3}\right)=\pi / 6 \times$ length $\times$ width $\times$ depth, and energy efficiency factor $(\mathrm{EEF})$ was measured according to the formula: $\operatorname{EEF}\left(\mathrm{J} / \mathrm{mm}^{3}\right)=$ $\eta \mathrm{Pt} / \mathrm{V}$, where $\eta$ (set to 0.7 ) means the focusing coefficient of HIFU transducer, P (W) means the power of HIFU, and $t(s)$ means the ablation time.

\section{Evaluation of Anticancer Efficiency of GVs-E. coli}

The antitumor efficiency was observed by measuring the body weight and tumor volume using an electronic caliper every 2 days from day 0 to day 21. Relative tumor volume (RTV) was normalized based on the initial tumor volume before the treatment. The tumor inhibition rate (IRT) was calculated by the formula:

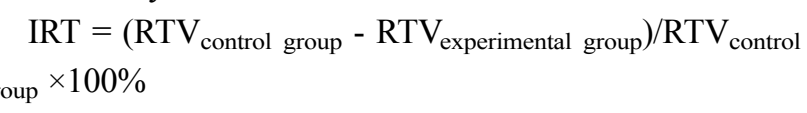

On the 21st day after injection, in order to observe apoptosis and necrosis, nude mice were euthanized and the tumor tissues were harvested for weighting and staining by $\mathrm{H} \& \mathrm{E}$, terminal-deoxynucleotidyl transferasemediated nick end labeling (TUNEL), and proliferating cell nuclear antigen (PCNA).

\section{Statistical Analysis}

All statistical analyses were performed using SPSS 21.0 program. Data are presented as mean \pm standard deviation (SD). Multi-group comparisons were analyzed using oneway ANOVA followed by LSD test or Dunnett's T3 test $(* \mathrm{p}<0.05, * * \mathrm{p}<0.01, * * * \mathrm{p}<0.001)$.

\section{Results and Discussion Characterization of $E$. coli $\mathrm{BL} 2 \mathrm{I}(\mathrm{Al})$}

To observe the basic characteristics of $E$. coli BL21(AI), the gram stain was performed. As Figure 2A shown, E. coli BL21(AI) shows a pale pink, long, and rod shape which was classified as a member of the gramnegative bacterium. E. coli BL21(AI) could grow rapidly under optimal conditions, and it reached the mid-logarithmic phase in 2-4 hours under the LB liquid 

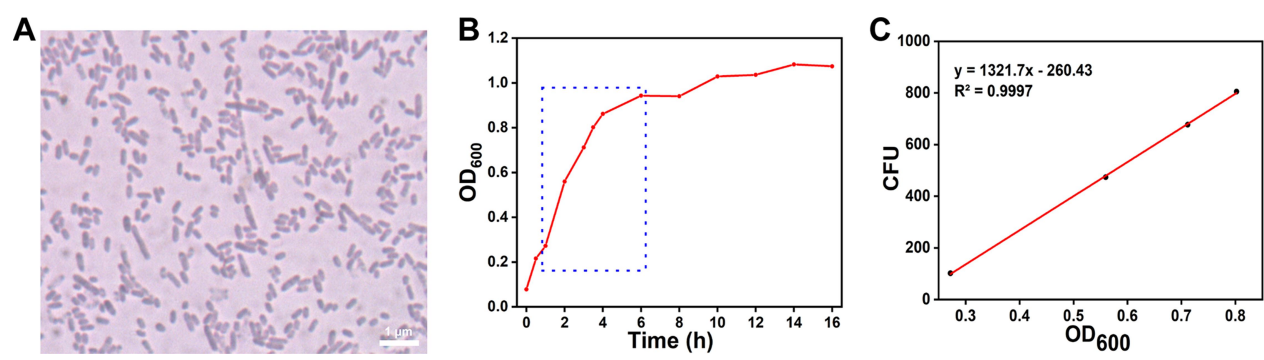

Figure 2 Characterization of $E$. coli $\mathrm{BL2I}(\mathrm{Al})$. (A) Optical microscope image of gram staining of $E$. coli $\mathrm{BL2I}(\mathrm{Al})(\mathrm{I} 000 \times$ magnification), the scale bar is I $\mu \mathrm{m}$. (B) Growth curve of $E$. coli $\mathrm{BL} 2 \mathrm{I}(\mathrm{Al})$ at $37^{\circ} \mathrm{C}, 220 \mathrm{rpm}$. (C) Bacterial counting corresponding to $\mathrm{OD}_{600}$.

Abbreviation: $\mathrm{OD}_{600}$, optical density $=600 \mathrm{~nm}$.

medium (dilution ratio: 1:100) in a shaking incubator with $220 \mathrm{rpm}$ at $37^{\circ} \mathrm{C}$ (Figure 2B). To quantify the E. coli BL21(AI), plate dilution was used to count the colony-forming units (CFU) by measuring absorbance value at $600 \mathrm{~nm}$ (optical density, $\mathrm{OD}_{600}$ ). According to the standard curve we calculated (Figure 2C) that the CFU was $1.06 \times 10^{9}$ in $1 \mathrm{~mL}$ of bacterial solutions when $\mathrm{OD}_{600}=1\left(1 \mathrm{OD}=1.06 \times 10^{9} \mathrm{CFU} / \mathrm{mL}\right)$.

\section{In vivo Biosafety Assay of $E$. coli BL2I(Al)}

The biosafety of bacteria is a prerequisite for use in vivo experiments and further clinical translation. As shown in Figure 3A, the bodyweight of mice exhibited a slight decrease 3 days after i.v. injection, but quickly showed an upward trend and the growth curve was consistent with the control group, indicating E. coli BL21(AI) treatment had no obvious effects on the body weight. The blood indexes including complete blood count (WBC, RBC, PLT, HGB, $\mathrm{MCH}$, and $\mathrm{MCV}$ ), liver functional markers (ALT, AST), myocardial enzymogram (CK, LDH-L), and kidney functional marker (BUN, CREA) showed slight fluctuations on day 1 after i.v. injection. At later time points (on day 3, 7 , or 14 days after injection), the blood indexes gradually return to the normal level compared with those in the control group, indicating undetectable toxicity in short and relatively long timeframes (Figure 3B). This result is consistent with the previous studies. ${ }^{47}$ The reason for the slight fluctuation of body weight and the blood indexes in the early time after i.v. injection might be sensitization of immune system, but this phenomenon can quickly disappear, because the $E$. coli BL21(AI) was cleared by normal organs. ${ }^{48} \mathrm{H} \& \mathrm{E}$ staining was further used to test the histocompatibility of $E$. coli
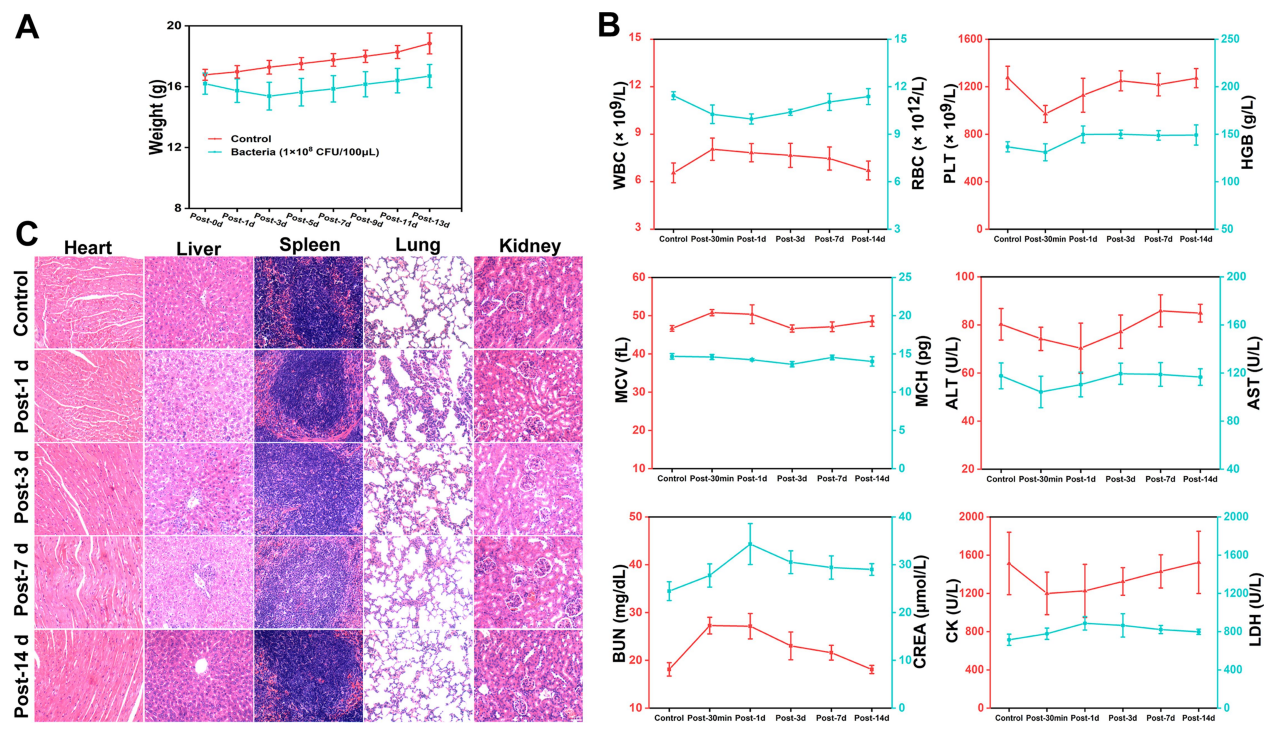

Figure 3 In vivo safety evaluation of $E$. coli BL2I(Al). (A) Bodyweight changes over time (B) Hematological assay of BALB/c mice and (C) H\&E staining of major organs from the control group and the experimental groups I, 3, 7, I4 days after i.v. injection of $E$. coli BL2I(AI). All the scale bars are $50 \mu \mathrm{m}$.

Abbreviations: H\&E, hematoxylin and eosin staining; i.v., intravenous. 
BL21(AI). H\&E staining of the major organs (heart, liver, spleen, lung, and kidney) at the corresponding time point after i.v. injection showed no significant acute or chronic physiological toxicity when compared with the control group (Figure $3 \mathrm{C}$ ), indicating the high histocompatibility of $E$. coli BL21(AI).

\section{Tumor-Targeting Ability of E. coli BL2I (Al)}

To elaborate the distribution of E. coli BL21(AI) in vivo after i.v. injection, the mice were executed, and the major organs including heart, liver, spleen, lung, kidney, and tumor tissues of mice were weighed, homogenized, and plated on LB plates. It can be observed that bacteria were gradually eliminated from major organs such as the heart, liver, spleen, kidney, and lung. In marked contrast, bacteria were colonized in tumor tissues (Figure 4A). By counting the CFU in each plate, it was found that the CFU in major organs including heart, liver, spleen, kidney, and lung gradually decreased during the time $(1,3,7,14$ days after injection). On the contrary, the CFU in tumor tissues increased exponentially following the time points (Figure 4B). Those results were consistent with the previous studies. ${ }^{49}$ This phenomenon may be attributed to the selected colonization of anaerobes in the hypoxic, immunosuppressive, and biochemically unique tumor microenvironment. ${ }^{50}$ The single colony on the plate was picked for gram staining. As shown in Figure $4 \mathrm{C}$, the pale pink, long, and rod-shaped bacteria can be observed, the same with Figure 2A, which indicated the bacteria growth in the plate is $E$. coli BL21(AI).

To further confirm whether GVs-E. coli could be expressed consistently in the tumor site, a green fluorescent protein (GFP) - bearing plasmid was transformed into E. coli BL21(AI) (GFP-E. coli). As shown in Figure 4D, strong green fluorescence was detected in the LB medium with or without ampicillin (Amp). On the contrary, the fluorescence intensity of GFP cultured in PBS was almost invisible, indicating that the target gene expressed in E. coli BL21(AI) could be expressed consistently in the optimum culture conditions. Moreover, to evaluate the ability of consistent expression of GFP-E. coli in vivo, the GFP-E. coli was injected into the MDA-MB-231 tumor-bearing mice intravenously. As shown in Figure $4 \mathrm{E}$, there was no green fluorescence detected in the control group. In marked contrast, green fluorescence was detected in the tumor tissues from day 1 to day 14 after i.v. injection of GFP-E.coli, demonstrating that not only GFP-E.coli could colonize in tumor tissues but also the plasmid would not vanish for a short time in vivo. ${ }^{51}$

\section{ARG Expressing in E. coli BL2I (Al)}

TEM was used to further validate whether the GVs was produced successfully in E. coli BL21(AI). A mass of cylindrical or spindle-shaped protein nanostructures can be directly observed in the E. coli BL21(AI) by TEM (Figure 5A), while none can be found in the control group (Figure 5B).

To evaluate the ultrasound imaging capability of GVs-E. coli, we investigated GVs-E. coli in vitro through a gel phantom (Figure 5C), and the corresponding quantitative analysis of gray value was performed (Figure 5D). Compared with those in the control group, both B-mode and CEUS-mode showed obvious echogenicity in GVs-E. coli $(* * * \mathrm{P}<0.001)$, demonstrating that GVs could be detected in vitro by ultrasound imaging. Next, an in vivo tumor ultrasound imaging experiment was performed to determine whether GVs-E. coli could be detected by ultrasound. We investigated the ultrasound imaging of tumors before and after injecting GVs-E. coli (Figure 5E), and the corresponding quantitative analysis of gray value was performed (Figure 5F). As the Figures are shown in Figure 5E and F, before injection, no echogenicity showed in both mode ultrasound images of tumors, and the gray value of tumor images had no obvious difference. In contrast, the echogenicity in both mode ultrasound was significantly enhanced after injecting GVs-E. coli $(* * * \mathrm{P}<0.001)$. The result of ultrasound imaging is consistent with those of previous studies, which confirmed that the GVs produced in E. coli as a contrast agent could be imaged by ultrasound. $^{52,53}$

\section{GVs-E. coli as HIFU Synergistic Agent by Inertial Cavitation in vitro}

Based on the distinct gas-filled protein nanoparticles property of GVs, their ex vivo synergistic effect on HIFU ablation was systematically investigated by employing fresh degassed bovine liver. As shown in Figure 6A, the HIFU ablation volume in all groups increased as adopted ultrasound power increased (120 $\mathrm{W}, 150 \mathrm{~W}$, and $180 \mathrm{~W})$. The gray-value from real-time ultrasound imaging of the GVs-E. coli group at three 

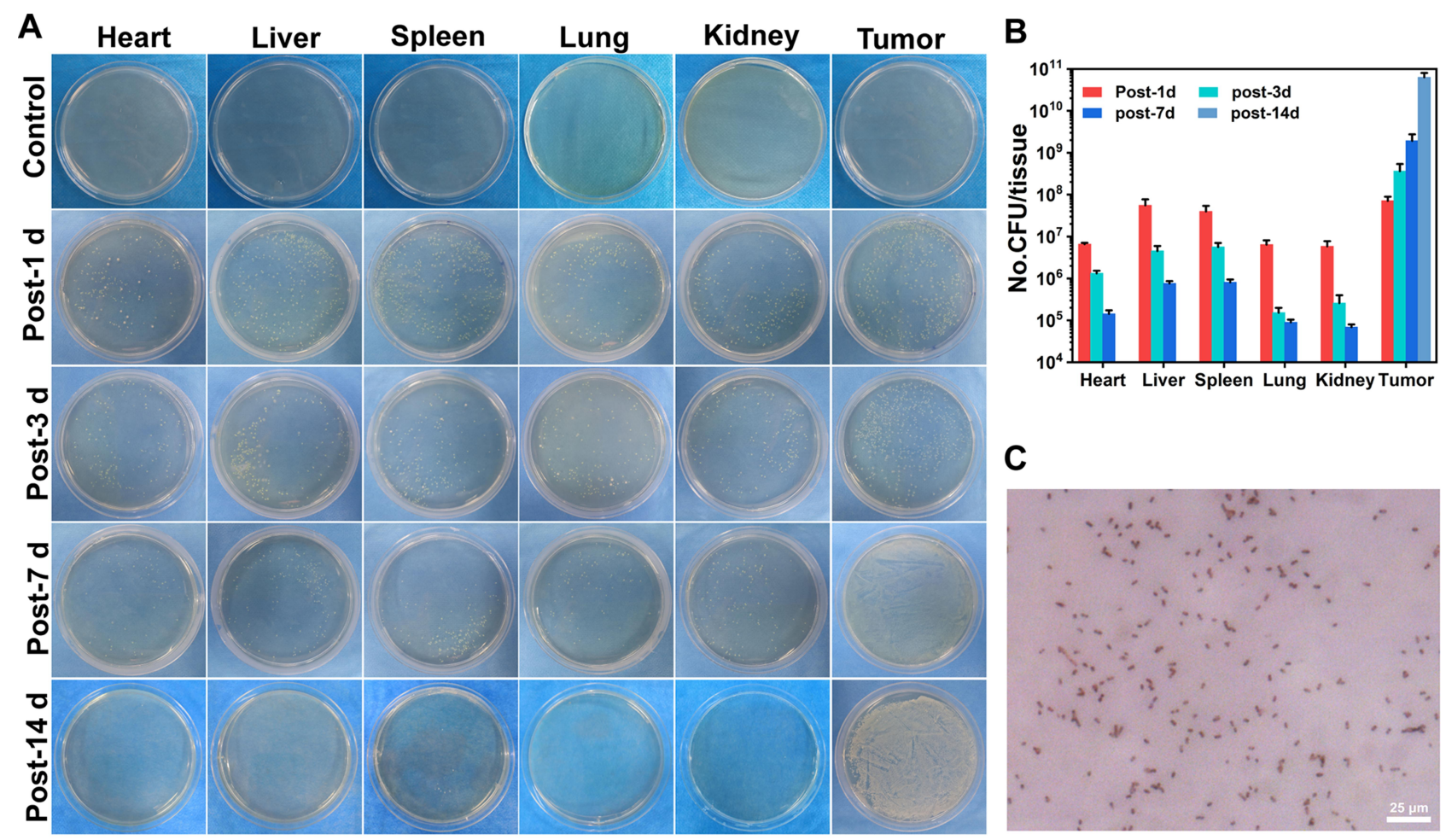

\section{C}
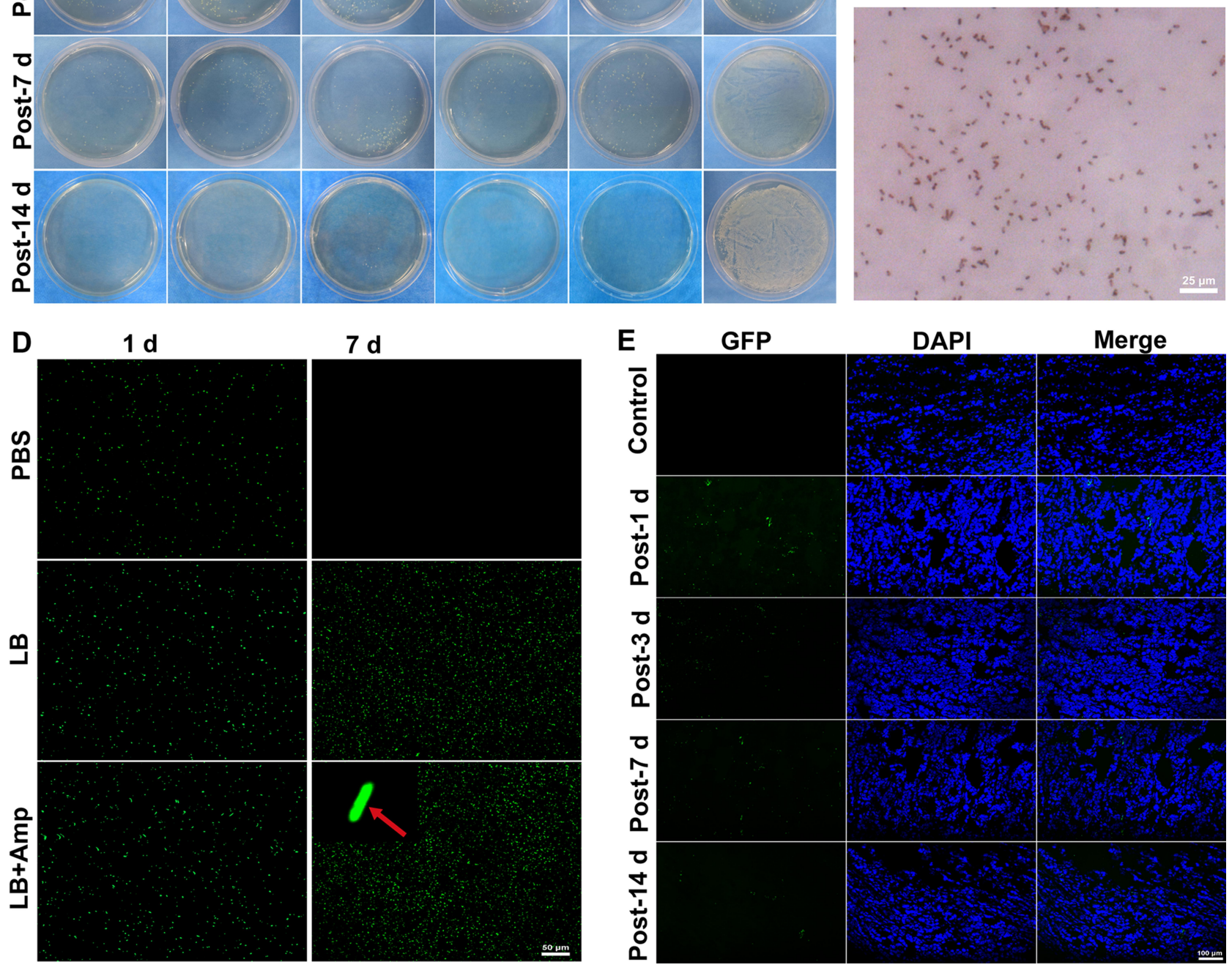

Figure 4 Bacterial colonization in MDA-MB-23I-bearing mice after i.v. injection. (A) Representative photographs of solid LB agar plates of bacterial colonization in various organs at different time points after injection of $E$. coli BL2I (Al). (B) Quantification of bacterial colonization in various organs of MDA-MB-23I-bearing mice at different time points after injection of $E$. coli BL2I (Al). (C) Optical microscope image of gram stain of a monoclonal colony from the LB plates (400 $\times$ magnification), the scale bar is $25 \mu \mathrm{m}$. (D) Representative CLSM images of GFP-E. coli under different culture conditions at $37{ }^{\circ} \mathrm{C}$, the scale bar is $50 \mu \mathrm{m}$. The red arrow marks the GFP-E. coli (I200 $\times$ magnification). (E) Representative CLSM images of GFP-E. coli growth in tumors at different time points after i.v. injection, the scale bar is $100 \mu \mathrm{m}$.

Abbreviations: i.v., intravenous; LB, Luria-Bertani; H\&E, hematoxylin and eosin staining; i.v., intravenous; GFP, green fluorescent protein.

levels of HIFU power was significantly higher than that in the other two groups (Figure $6 \mathrm{~B}, * \mathrm{p}<0.05$ ). Similarly, the specific volume of coagulative necrosis of the GVs- $E$. coli group was significantly larger than that in the other two groups (Figure $6 \mathrm{C}, * \mathrm{p}<0.05$ ). At the same time, there were no obvious broadband 

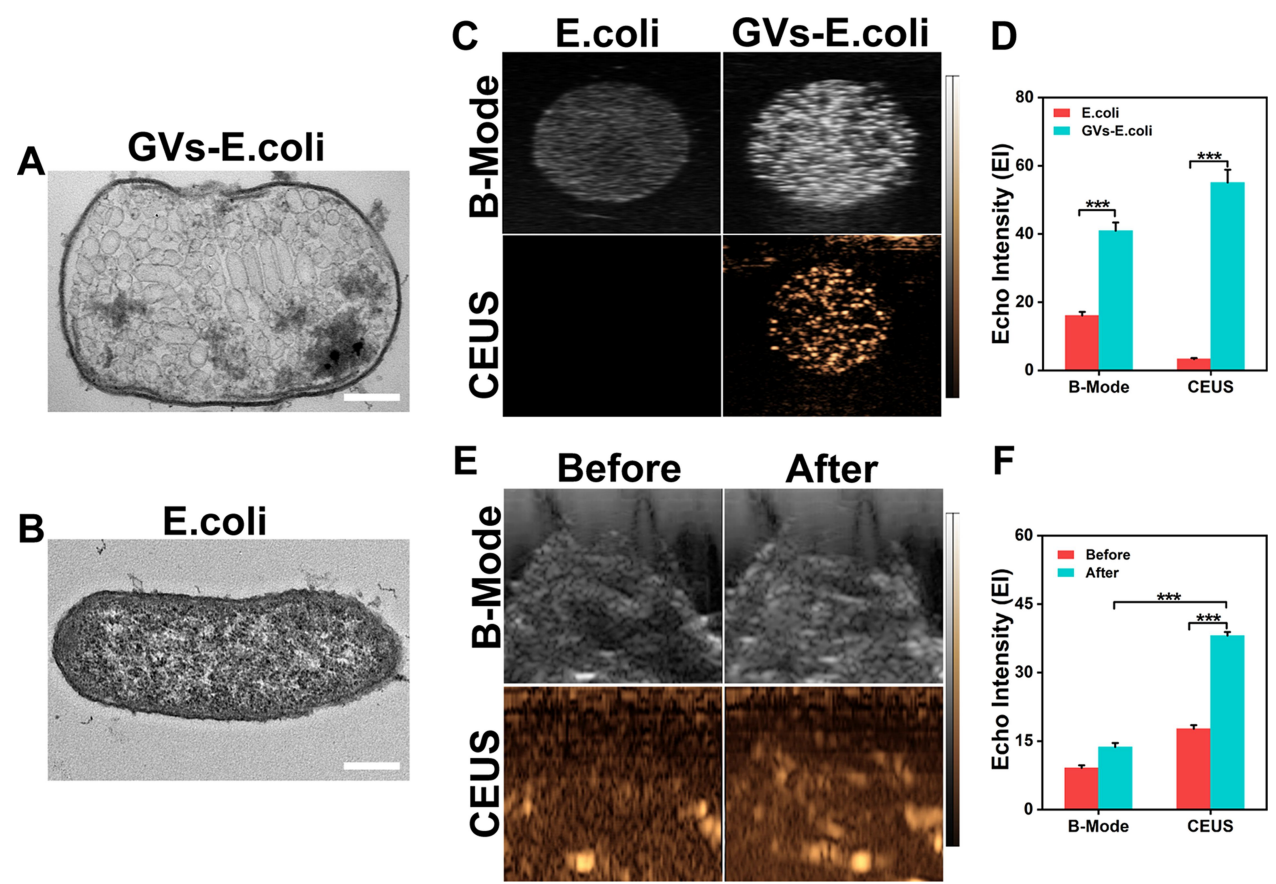

$\mathbf{F}$

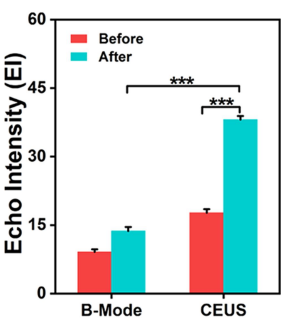

Figure 5 ARG expression in E. coli BL2I(AI). (A, B) Representative TEM images of GVs-E. coli and E. coli, the scale bar is $500 \mathrm{~nm}$. (C) In vitro ultrasound images of $E$. coli and GVs-E. coli in B-mode and CEUS. (D) Quantitative analysis of echo intensity in B-mode and CEUS ( $\mathrm{n}=3$, *** $\mathrm{p}<0.00 \mathrm{I})$. (E) In vivo ultrasound images of tumors before and after injecting GVs-E. coli in B-mode and CEUS. (F) Quantitative analysis of echo intensity in B-mode and CEUS ( $n=3$, *** $p<0.001$ ).

Abbreviations: ARG, acoustic reporter gene; TEM, transmission electron microscopy; E. coli, Escherichia coli; GVs, gas vesicles; CEUS, contrast-enhanced ultrasonography.

emissions detected during the ultrasound power (120 $\mathrm{W}, 150 \mathrm{~W}$, and $180 \mathrm{~W}$ ) in PBS groups (Figure 6D1, D4, and D7) and E. coli groups (Figure 6D2, D5, and D8). Notably, the broadband emissions of GVs-E. coli group showed a significant increase in detected signal amplitude as the power increased (Figure 6D3, D6, and D9). These results suggest that GVs-E.coli was able to improve HIFU-ablation efficacy by serving as nuclei for inertial cavitation. ${ }^{42}$

\section{Anticancer Efficiency of GVs-E. coli Induced by Combination Therapy}

To further evaluate the anticancer efficiency of GVsE. coli, the synergistic effect of HIFU ablation and tumor growth inhibition through GVs-E. coli were systematically assessed. Figure 7A shows the protocol of this study, HIFU treatment was conducted on 7 days after i.v. injection of GVs-E. coli. As the results are shown in Figure 7B, after HIFU irradiation, the gray value of the tumor target region in all groups (PBS+HIFU, E. coli $+\mathrm{HIFU}$, and GVs-E. coli $+\mathrm{HIFU})$ showed obvious changes compared with the result before HIFU irradiation. Notably, the gray value changes in the GVs-E. coli group were more significant than those in the other two groups (Figure 7B and C, $* * * p<0.001)$. At the same time, we detected the cavitation effect by PCD. The GVs-E.coli group showed obviously higher broadband emissions than the other two groups (Figure 7D), indicating GVsE. coli could serve as nuclei for inertial cavitation. ${ }^{42}$ At $24 \mathrm{~h}$ after HIFU irradiation, the tumor tissue was harvested for TTC staining and measuring the ablation volumes. As shown in Figure 7E, the coagulative necrosis volume was gray-white (arrowheads indicate points), and the non-ablated regions were red. The gray-white area in the GVs-E. coli group was larger than the other two groups, and the corresponding quantitative coagulative necrosis volume showed the same results as the result of gray value (Figure $7 \mathrm{~F}, * * * \mathrm{p}<$ 0.001). Moreover, the value of EEF in various groups showed an opposite trend compared with coagulative necrosis volume and gray value (Figure $7 \mathrm{G}, * * \mathrm{p}<$ 0.01 ), which means ultrasonic energy for ablating a unit volume of the tumor is the least when using GVs-E. coli as the SA, indicating that GVs-E. coli could be used as a SA for HIFU therapy, and 

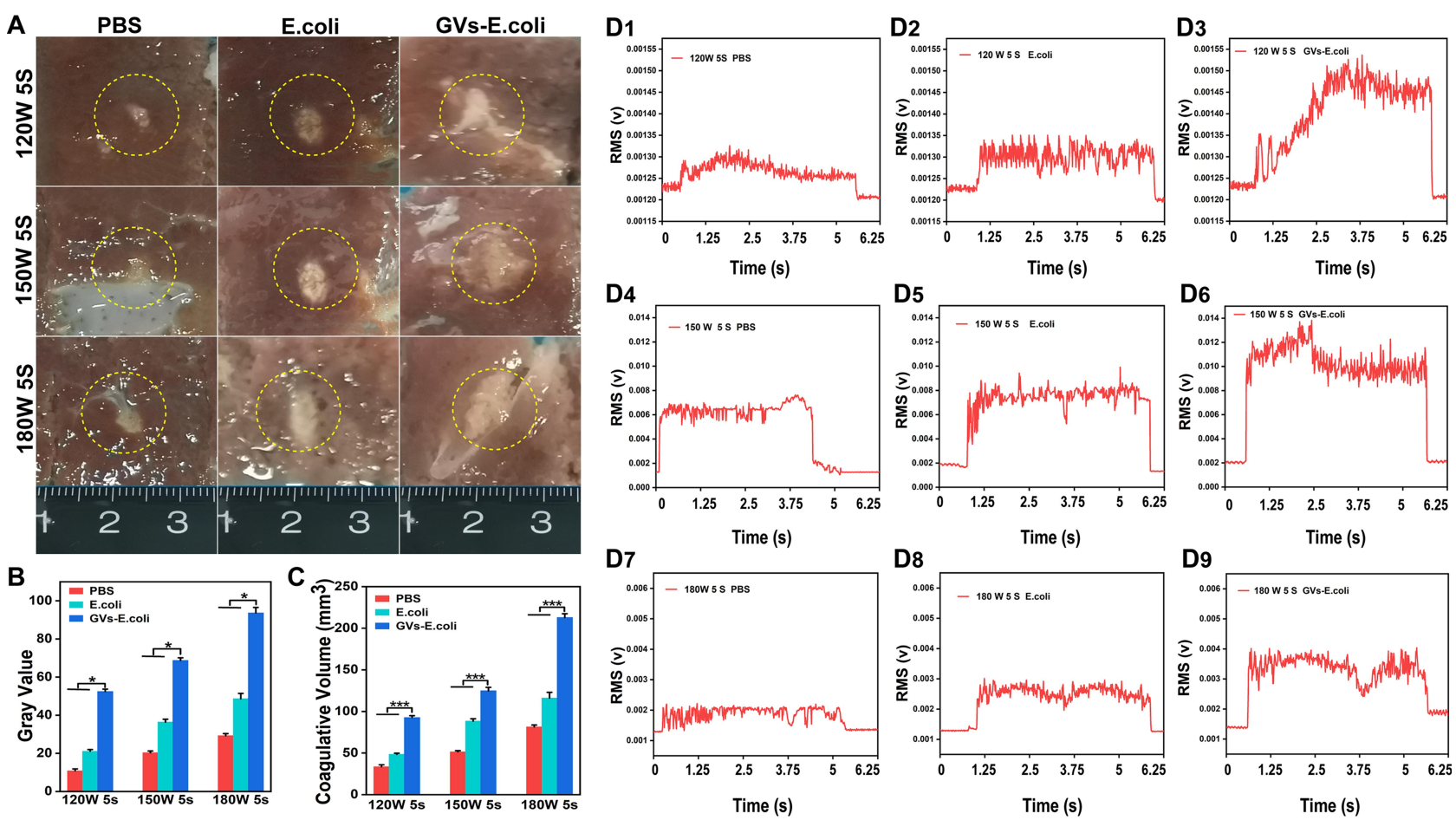

Figure 6 Synergistic HIFU therapy of GVs-E. coli in vitro. (A) Representative photographs of ablated bovine livers after HIFU irradiation. The yellow dotted circles marks the coagulative necrosis of bovine livers). (B) The corresponding quantitative analysis of the gray value of bovine livers after HIFU irradiation ( $n=3$, *p $<0.05)$. (C) The corresponding quantitative analysis of coagulation volumes of bovine livers after HIFU irradiation ( $n=3$, *** $<<0.00 \mathrm{I})$. (DI-D9) Representative images of cavitation activity at the HIFU irradiation in different power for PCD signals.

Abbreviations: E. coli, Escherichia coli; GVs, gas vesicles; CEUS, contrast-enhanced ultrasonography; PCD, passive cavitation detector; HIFU, high-intensity focused ultrasound.

synergized HIFU ablation mainly owing to the cavitation effect.

To further investigate the combination therapeutic effect on tumors, we monitored body weight and the tumor volume changes every 2 days from day 0 to day 21 after i.v. injection (Figure 8A). The bodyweight of mice in the experimental groups except for the HIFU group exhibited a slight decrease in 3 days after i.v. injection, but quickly showed an upward trend and the growth curve was consistent with the control group (Figure 8B), indicating that the combination therapy had no effects on the body weight. As shown in Figure 8C, a significant difference of tumor growth inhibition in all experimental groups was observed compared with the control group, and GVsE. coli + HIFU group showed the greatest tumor growth inhibition during the observed time $(* * * p<0.001)$. Moreover, Figure 8D showed that the tumor inhibition rate $(87 \%)$ of the GVs-E. coli + HIFU group was much higher than that of the HIFU group (75\%), E. coli group (66\%), GVs-E. coli group (64\%), and E. coli + HIFU
$(77 \%)(* * * p<0.001)$. Furthermore, tumor weight was consistent with that of the variation of tumor volume (insert: digital photos, Figure $8 \mathrm{E})(* * * \mathrm{p}<0.001)$, wherein the GVsE. coli + HIFU group acquired the lowest tumor weight. These results confirmed that $E$. coli possess the ability to inhibit tumor growth and the mechanism behind this result is mainly due to: i) bacterial toxins that directly kill cancer cells; ii) cytokines that stimulate immune cells to kill cancer cells; iii) tumor antigens that sensitize the immune system against cancer cells. ${ }^{43}$ The primary mechanism of HIFU ablation for tumors is the focusing of the ultrasound beam on a point to generate high energy through the thermal effect, cavitation effect, and mechanical effect, which can stimulate protein denaturation and coagulation necrosis of tumor tissues without affecting surrounding normal tissue, thereby achieving non-invasive cancer treatment. $^{54} \mathrm{GVs}-E$. coli showed the strongest synergistic effects mainly due to the cavitation effect. In this study, the GVs-E. coli + HIFU group showed more effective cancer treatment because the $E$. coli could first inhibit 


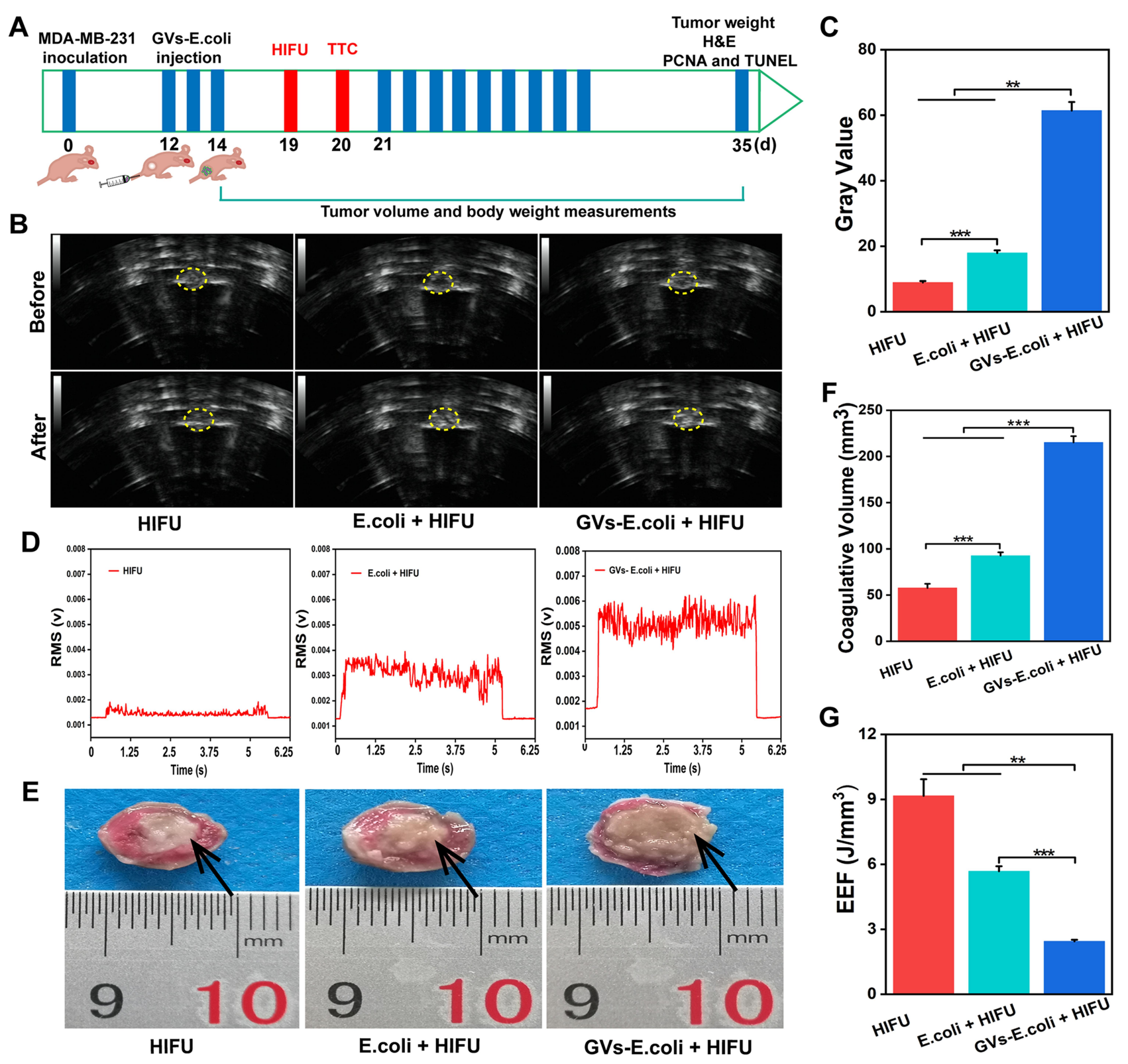

Figure 7 Synergistic effect of GVs-E. coli by HIFU ablation. (A) The schematic illustration of the treatment protocol. (B) In vivo ultrasound imaging of tumor tissues (yellow circle) before HIFU ablation and after HIFU ablation in a different group. The yellow dotted circle marks the tumor nodules. (C) The comparison of gray values of tumor tissues in each group after HIFU irradiation $(n=5, * * P<0.05, * * * P<0.00 I)$. (D) Representative images of cavitation activity at the HIFU irradiation for PCD signals. ( $E$ ) Coagulative necrosis of tumors by TTC staining after HIFU therapy, the necrotic tissue appears gray and the normal tumor tissue is red (black arrows marks the necrotic tissue). (F) The comparison of coagulative necrosis of tumor tissues in each group after HIFU irradiation ( $n=5$, $* * * P<0.00 \mathrm{I})$. (G) The comparison of EEF of tumor tissues in each group after HIFU Irradiation ( $\mathrm{n}=5, * * \mathrm{P}<0.05$, ***P $<0.00 \mathrm{I})$.

Abbreviations: $E$. coli, Escherichia coli; GVs, gas vesicles; HIFU, high-intensity focused ultrasound; PCD, passive cavitation detector; TTC, 2,3,5-triphenyltetrazolium chloride; EEF, energy efficiency factor.

the tumor growth and then avoid liquefactive necrosis of the tumor, thus facilitating the HIFU ablation for solid tumor tissues.

In addition, the combination therapy effect was assessed via histological analyses such as hematoxylin and eosin (H\&E), PCNA, and TUNEL staining. As shown in Figure 8F, a clear boundary between the ablated area and the non-ablated area was observed, and substantial cellular necrosis was observed in GVs-E. coli + HIFU group. Lower proliferative tumor cells were determined by PCNA assay and extensive apoptotic nuclei were observed using TUNEL assay (Figure 8G), demonstrating that the combination therapy can achieve more effective cancer treatment than that of the monotherapies. 
A

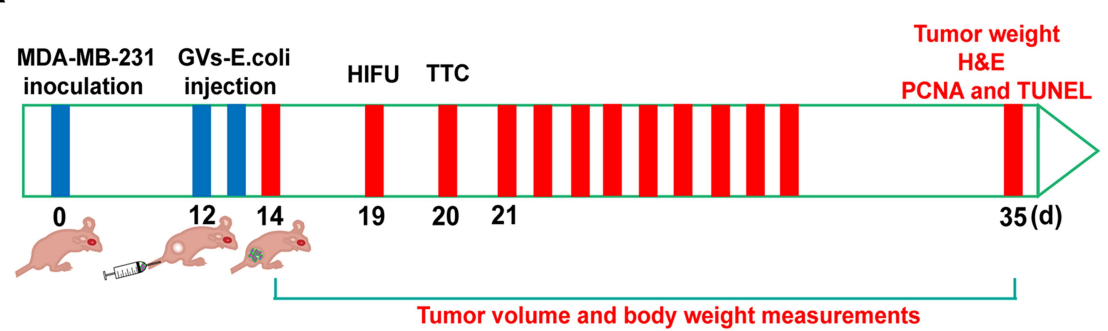

C
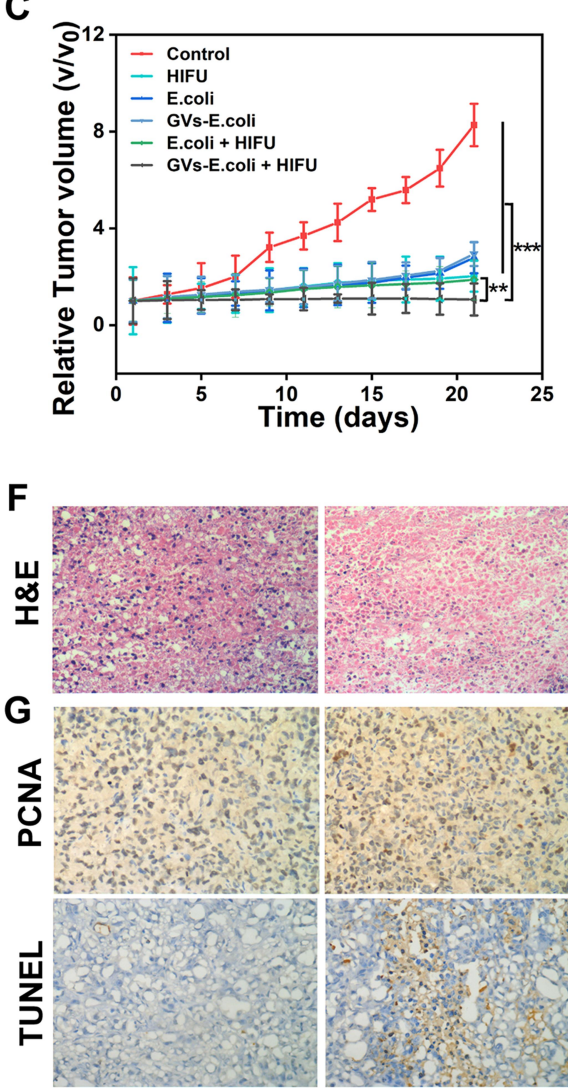

Control
D

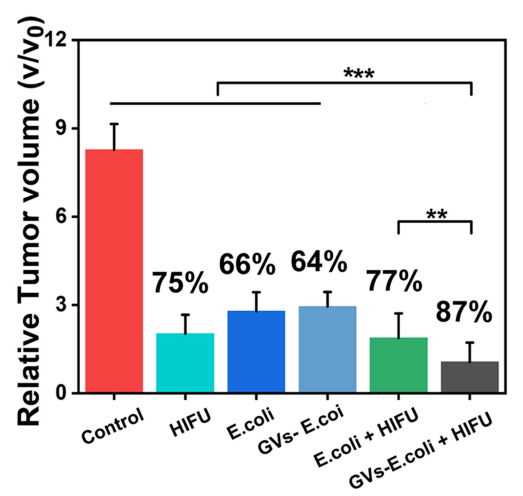

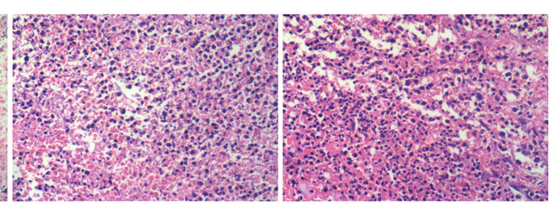

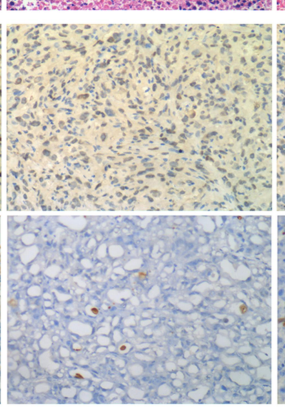

E.coli

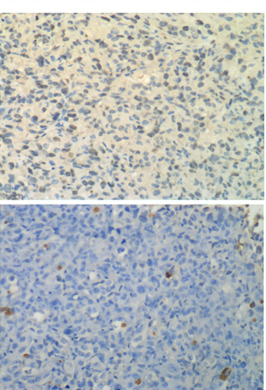

GVs-E.coli
B

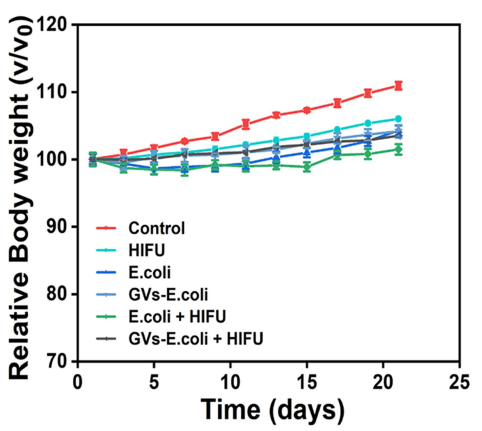

E

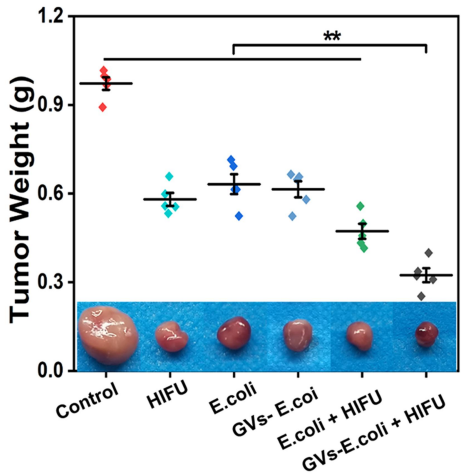

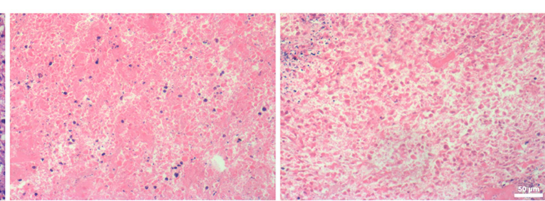

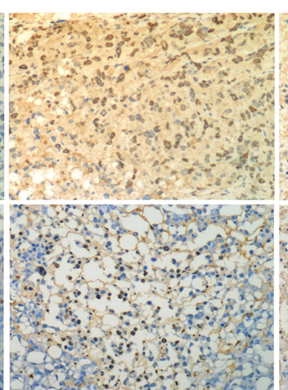

E.coli + HIFU

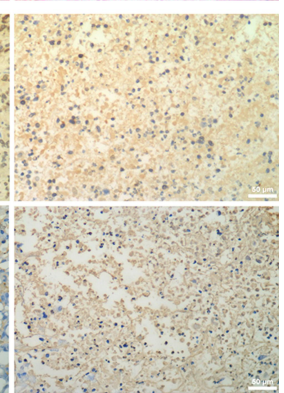

GVs-E.coli + HIFU

Figure 8 The anticancer effect of combination therapy. (A) The schematic illustration of the treatment protocol. (B) Bodyweight changes over time. (C) Tumor volume of different groups $(n=5, * * P<0.01$, ***P<0.00I). (D) IRT of different groups $(n=5, * * P<0.01$, ***P $<0.00 I)$. (E) Tumor weight on the 2 I st day (Insert: digital photos of tumors on the 2 Ist day) $(n=5, * * P<0.0 I)$. (F) Optical microscope images of tumor sections from each group after HIFU ablation were stained for HE staining. (G) PCNA and TUNEL staining of tumor sections from various treatment groups for observing proliferation and apoptosis. All the scale bar is $50 \mu \mathrm{m}$.

Abbreviations: PCNA, proliferating cell nuclear antigen; TUNEL, apoptosis expression.

\section{Conclusion}

In summary, we have treated breast cancer more efficiently by taking the novel strategy of combing bacteriotherapy with HIFU therapy. Detailly, we successfully expressed ARG in E. coli to produce GVs, which is gas-containing protein nanoparticles and could be imaged by ultrasound in vitro and in vivo. The GVs-E. coli exhibited excellent biosafety at a safe tolerated dose. We demonstrated that GVs-E. coli can specifically target the tumor site and inhibit the tumor growth to avoid liquefactive necrosis of the tumor, which is more conducive to HIFU ablation for solid tumors. Moreover, the genetic reconstitution of bacteria protein nanoparticles (GVs-E. coli) could act as the cavitation nuclei to synergize HIFU therapy efficiently. Therefore, GVs-E. coli could act as a potential biological targeting synergistic agent to synergize HIFU therapy for cancers more efficiently. This novel combination strategy also shows great potential in fighting cancers. 


\section{Acknowledgments}

We greatly acknowledge the help and selfless sharing from Dr. Mikhail Shapiro and Dr. Zhiyang Jin.

\section{Disclosure}

The authors report no conflicts of interest in this work.

\section{References}

1. Cai Y, Liang P, Tang Q, et al. Diketopyrrolopyrrole-Triphenylamine Organic Nanoparticles as Multifunctional Reagents for Photoacoustic Imaging-Guided Photodynamic/Photothermal Synergistic Tumor Therapy. ACS Nano. 2017;11(1):1054-1063. doi:10.1021/ acsnano.6b07927

2. Zhou S, Gravekamp C, Bermudes D, Liu K. Tumour-targeting bacteria engineered to fight cancer. Nat Rev Cancer. 2018;18 (12):727-743. doi:10.1038/s41568-018-0070-z

3. Zhang C, Sun W, Wang Y, et al. Gd-/CuS-Loaded Functional Nanogels for MR/PA Imaging-Guided Tumor-Targeted Photothermal Therapy. ACS Appl Mater Interfaces. 2020;12 (8):9107-9117. doi:10.1021/acsami.9b23413

4. Zhou Y, Hu Y, Sun W, et al. Polyaniline-loaded $\gamma$-polyglutamic acid nanogels as a platform for photoacoustic imaging-guided tumor photothermal therapy. Nanoscale. 2017;9(34):12746-12754. doi: $10.1039 / \mathrm{c} 7 \mathrm{nr} 04241 \mathrm{~h}$

5. Cheng C, Chen W, Zhang L, Wu H, Zink J, Responsive Mesoporous A. Silica Nanoparticle Platform for Magnetic Resonance Imaging-Guided High-Intensity Focused Ultrasound-Stimulated Cargo Delivery with Controllable Location, Time, and Dose. J Am Chem Soc. 2019;141 (44):17670-17684. doi:10.1021/jacs.9b07591

6. Kang Y, Kim J, Park J, et al. Tumor vasodilation by N-Heterocyclic carbene-based nitric oxide delivery triggered by high-intensity focused ultrasound and enhanced drug homing to tumor sites for anti-cancer therapy. Biomaterials. 2019;217:119297. doi:10.1016/j. biomaterials.2019.119297

7. Wang Z, Qiao R, Tang N, et al. Active targeting theranostic iron oxide nanoparticles for MRI and magnetic resonance-guided focused ultrasound ablation of lung cancer. Biomaterials. 2017;127:25-35. doi:10.1016/j.biomaterials.2017.02.037

8. Liang X, Gao J, Jiang L, et al. Nanohybrid liposomal cerasomes with good physiological stability and rapid temperature responsiveness for high intensity focused ultrasound triggered local chemotherapy of cancer. ACS Nano. 2015;9(2):1280-1293. doi:10.1021/nn507482w

9. Yildirim A, Chattaraj R, Blum N, Goldscheitter G, Goodwin A. Stable Encapsulation of Air in Mesoporous Silica Nanoparticles: fluorocarbon-Free Nanoscale Ultrasound Contrast Agents. $A d v$ Healthcare Mater. 2016;5(11):1290-1298. doi:10.1002/adhm.201600030

10. Wang S, Zhao J, Hu F, et al. Phase-changeable and bubble-releasing implants for highly efficient HIFU-responsive tumor surgery and chemotherapy. J Mater Chem B. 2016;4(46):7368-7378. doi:10.1039/c6tb01861k

11. Liu T, Zhang N, Wang Z, et al. Endogenous Catalytic Generation of O Bubbles for In Situ Ultrasound-Guided High Intensity Focused Ultrasound Ablation. ACS Nano. 2017;11(9):9093-9102. doi:10.1021/acsnano.7b03772

12. You Y, Wang Z, Ran H, et al. Nanoparticle-enhanced synergistic HIFU ablation and transarterial chemoembolization for efficient cancer therapy. Nanoscale. 2016;8(7):4324-4339. doi:10.1039/ c5nr08292g

13. Moncion A, Harmon J, Li Y, et al. Spatiotemporally-controlled transgene expression in hydroxyapatite-fibrin composite scaffolds using high intensity focused ultrasound. Biomaterials. 2019;194:14-24. doi:10.1016/j.biomaterials.2018.12.011
14. Duong M, Qin Y, You S, Min J. Bacteria-cancer interactions: bacteria-based cancer therapy. Exp Mol Med. 2019;51(12):1-15. doi:10.1038/s12276-019-0297-0

15. Kaimala S, Al-Sbiei A, Cabral-Marques O, Fernandez-Cabezudo M, Attenuated A-RB. Bacteria as Immunotherapeutic Tools for Cancer Treatment. Front Oncol. 2018;8:136. doi:10.3389/fonc.2018.00136

16. Sedighi M, Zahedi Bialvaei A, Hamblin M, et al. Therapeutic bacteria to combat cancer; current advances, challenges, and opportunities. Cancer Med. 2019;8(6):3167-3181. doi:10.1002/ cam4.2148

17. Yoo J, Irvine D, Discher D, Mitragotri S. Bio-inspired, bioengineered and biomimetic drug delivery carriers. Nat Rev Drug Discov. 2011;10 (7):521-535. doi:10.1038/nrd3499

18. Sivan A, Corrales L, Hubert N, et al. Commensal Bifidobacterium promotes antitumor immunity and facilitates anti-PD-L1 efficacy. Science. 2015;350(6264):1084-1089. doi:10.1126/science.aac4255

19. Yazawa K, Fujimori M, Nakamura T, et al. Bifidobacterium longum as a delivery system for gene therapy of chemically induced rat mammary tumors. Breast Cancer Res Treat. 2001;66(2):165-170. doi:10.1023/a:1010644217648

20. Danino T, Lo J, Prindle A, Hasty J, Bhatia S. In Vivo Gene Expression Dynamics of Tumor-Targeted Bacteria. ACS Synth Biol. 2012;1(10):465-470. doi:10.1021/sb3000639

21. Guo Y, Chen Y, Liu X, Min J, Tan W, Zheng J. Targeted cancer immunotherapy with genetically engineered oncolytic Salmonella typhimurium. Cancer Lett. 2020;469:102-110. doi:10.1016/j. canlet.2019.10.033

22. Chen W, Wang Y, Qin M, et al. Bacteria-Driven Hypoxia Targeting for Combined Biotherapy and Photothermal Therapy. ACS Nano. 2018;12(6):5995-6005. doi:10.1021/acsnano.8b02235

23. Min J, Kim H, Park J, et al. Noninvasive real-time imaging of tumors and metastases using tumor-targeting light-emitting Escherichia coli. Mol Imaging Biol. 2008;10(1):54-61. doi:10.1007/s11307-007-0120-5

24. Zheng D, Chen Y, Li Z, et al. Optically-controlled bacterial metabolite for cancer therapy. Nat Commun. 2018;9(1):1680. doi:10.1038/ s41467-018-03233-9

25. Yu YA, Shabahang S, Timiryasova TM, et al. Visualization of tumors and metastases in live animals with bacteria and vaccinia virus encoding light-emitting proteins. Nat Biotechnol. 2004;22 (3):313-320. doi:10.1038/nbt937

26. Kim S, Castro F, Paterson Y, Gravekamp C. High efficacy of a Listeria-based vaccine against metastatic breast cancer reveals a dual mode of action. Cancer Res. 2009;69(14):5860-5866. doi:10.1158/0008-5472.can-08-4855

27. Chagnon A, Hudon C, McSween G, Vinet G, Fredette V. Cytotoxicity and reduction of animal cell growth by Clostridium M-55 spores and their extracts. Cancer. 1972;29(2):431-434. doi:10.1002/1097-0142(197202)29:2<431::AID-CNCR2820290226>3.0.CO;2-Z

28. Shinnoh M, Horinaka M, Yasuda T, et al. Clostridium butyricum MIYAIRI 588 shows antitumor effects by enhancing the release of TRAIL from neutrophils through MMP-8. Int J Oncol. 2013;42 (3):903-911. doi:10.3892/ijo.2013.1790

29. Yu X, Lin C, Yu J, Qi Q, Wang Q. Bioengineered Escherichia coli Nissle 1917 for tumour-targeting therapy. Microb Biotechnol. 2020;13(3):629-636. doi:10.1111/1751-7915.13523

30. Luo Y, Xu D, Gao X, et al. Nanoparticles conjugated with bacteria targeting tumors for precision imaging and therapy. Biochem Biophys Res Commun. 2019;514(4):1147-1153. doi:10.1016/j.bbrc.2019.05.074

31. Chen C, Wang Y, Tang Y, et al. Bifidobacterium-mediated high-intensity focused ultrasound for solid tumor therapy: comparison of two nanoparticle delivery methods. Int $j$ Hyperther. 2020;37 (1):870-878. doi:10.1080/02656736.2020.1791365

32. Xu D, Zou W, Luo Y, et al. Feasibility between Bifidobacteria Targeting and Changes in the Acoustic Environment of tumor Tissue for Synergistic HIFU. Sci Rep. 2020;10(1):7772. doi:10.1038/s41598-020-64661-6 
33. Wang Y, Chen C, Luo Y, et al. Experimental Study of Tumor Therapy Mediated by Multimodal Imaging Based on a Biological Targeting Synergistic Agent. Int $J$ Nanomedicine. 2020;15:1871-1888. doi:10.2147/ijn.s238398

34. Gao X, Zou W, Jiang B, et al. Experimental Study of Retention on the Combination of Bifidobacterium with High-Intensity Focused Ultrasound (HIFU) Synergistic Substance in Tumor Tissues. Sci Rep. 2019;9:1.

35. Bourdeau R, Lee-Gosselin A, Lakshmanan A, et al. Acoustic reporter genes for noninvasive imaging of microorganisms in mammalian hosts. Nature. 2018;553(7686):86-90. doi:10.1038/nature25021

36. Shapiro M, Goodwill P, Neogy A, et al. Biogenic gas nanostructures as ultrasonic molecular reporters. Nat Nanotechnol. 2014;9 (4):311-316. doi:10.1038/nnano.2014.32

37. Szablowski J, Bar-Zion A, Achieving Spatial SM. Molecular Specificity with Ultrasound-Targeted Biomolecular Nanotherapeutics. Acc Chem Res. 2019;52(9):2427-2434. doi:10.1021/acs.accounts.9b00277

38. Maresca D, Lakshmanan A, Lee-Gosselin A, et al. Nonlinear ultrasound imaging of nanoscale acoustic biomolecules. Appl Phys Lett. 2017;110(7):073704. doi:10.1063/1.4976105

39. Lakshmanan A, Lu G, Farhadi A, et al. Preparation of biogenic gas vesicle nanostructures for use as contrast agents for ultrasound and MRI. Nat Protoc. 2017;12(10):2050-2080. doi:10.1038/ nprot.2017.081

40. Lakshmanan A, Jin Z, Nety S, et al. Acoustic biosensors for ultrasound imaging of enzyme activity. Nat Chem Biol. 2020;16 (9):988-996. doi:10.1038/s41589-020-0591-0

41. Ling B, Lee J, Maresca D, et al. Biomolecular Ultrasound Imaging of Phagolysosomal Function. ACS Nano. 2020;14(9):12210-12221. doi:10.1021/acsnano.0c05912

42. Bar-Zion A, Nourmahnad A, Mittelstein DR, et al. Acoustically Detonated Biomolecules for Genetically Encodable Inertial Cavitation. bioRxiv. 2019:620567. doi:10.1101/620567.

43. Forbes N. Engineering the perfect (bacterial) cancer therapy. Nat Rev Cancer. 2010;10(11):785-794. doi:10.1038/nrc2934

44. Ho C, Tan H, Chua K, et al. Engineered commensal microbes for diet-mediated colorectal-cancer chemoprevention. Nat Biomed Eng. 2018;2(1):27-37. doi:10.1038/s41551-017-0181-y
45. Jiang S, Phan T, Nam T, et al. Inhibition of Tumor Growth and Metastasis by a Combination of Escherichia coli-mediated Cytolytic Therapy and Radiotherapy. Mol Ther. 2010;18 (3):635-642. doi:10.1038/mt.2009.295

46. Zhang Y, Zhang Y, Xia L, et al. Escherichia coli Nissle 1917 targets and restrains mouse B16 melanoma and 4T1 breast tumors through expression of azurin protein. Appl Environ Microbiol. 2012;78 (21):7603-7610. doi:10.1128/aem.01390-12

47. Zhang H, Man J, Liang B, et al. Tumor-targeted delivery of biologically active TRAIL protein. Cancer Gene Ther. 2010;17(5):334-343. doi:10.1038/cgt.2009.76

48. Sznol M, Lin SL, Bermudes D, Zheng L, King I. Use of preferentially replicating bacteria for the treatment of cancer. J Clin Invest. 2000;105(8):1027-1030.

49. Min J, Nguyen V, Kim H, Hong Y, Choy H. Quantitative bioluminescence imaging of tumor-targeting bacteria in living animals. Nat Protoc. 2008;3(4):629-636. doi:10.1038/nprot.2008.32

50. Kasinskas RW, Forbes NS. Salmonella typhimurium lacking ribose chemoreceptors localize in tumor quiescence and induce apoptosis. Cancer Res. 2007;67(7):3201-3209.

51. Zhang Y, Zhang Y, Xia L, et al. Escherichia coli Nissle 1917 targets and restrains mouse B16 melanoma and 4T1 breast tumors through expression of azurin protein. Appl Environ Microbiol. 2012;78 (21):7603.

52. Ling B, Lee J, Maresca D, et al. Biomolecular Ultrasound Imaging of Phagolysosomal Function. ACS Nano. 2020;14(9):12210-12221. doi:10.1021/acsnano.0c05912

53. Lakshmanan A, Jin Z, Nety SP, Sawyer DP, Shapiro MG. Acoustic biosensors for ultrasound imaging of enzyme activity. Nat Chem Biol. 2020;16(9):1-9.

54. Ma X, Yao M, Shi J, et al. High Intensity Focused Ultrasound-Responsive and Ultrastable Cerasomal Perfluorocarbon Nanodroplets for Alleviating Tumor Multidrug Resistance and Epithelial-Mesenchymal Transition. ACS Nano. 2020. doi:10.1021/ acsnano.0c07287
International Journal of Nanomedicine

\section{Publish your work in this journal}

The International Journal of Nanomedicine is an international, peerreviewed journal focusing on the application of nanotechnology in diagnostics, therapeutics, and drug delivery systems throughout the biomedical field. This journal is indexed on PubMed Central, MedLine, CAS, SciSearch ${ }^{\circledR}$, Current Contents ${ }^{\circledR} /$ Clinical Medicine,
Journal Citation Reports/Science Edition, EMBase, Scopus and the Elsevier Bibliographic databases. The manuscript management system is completely online and includes a very quick and fair peer-review system, which is all easy to use. Visit http://www.dovepress.com/ testimonials.php to read real quotes from published authors. 